\title{
On the Thompson-Wolf experiment: a study with laser sources
}

\section{David Paredes Barato}

\section{Maria L. Calvo}

mlcalvo@fis.ucm.es

\author{
Department of Optics, Faculty of Physics, Complutense University of Madrid
}

Department of Optics, Faculty of Physics, Complutense University of Madrid

We present a revisited study of the Thompson-Wolf experiment earlier developed in 1957 with the aim to characterize the degree of spatial coherent of a luminous source. We develop further experiments by using laser sources under various modal regimes. We analyze the experimental results. [DOI: 10.2971/jeos.2010.10051S]

Keywords: theory of partial coherence, laser sources, degree of spatial coherence

\section{INTRODUCTION}

${ }^{1}$ The invention of the laser in 1960 gave birth to a fifty years era of fascinating progress. At present, the dramatic development of new laser sources has configured a very rich technological map in which an enormous amount of basic and applied research and associated technologies are present in all fields of science. We will not enter in this article on the historical aspects of the invention of the laser, for obvious reasons. Other authors have contributed to an enlargement of the knowledge of how the sources working under the stimulated emission of radiation, for both visual and infrared frequencies, started in the early 60's and the previous theoretical approach as established by Albert Einstein in 1917 with his work on the quantum theory of radiation $[1,2]$.

A remarkable fact on the development of key concepts in the physics of the 20th C. is that the concept of photon was inside the very dual nature of light, involving the radiative interactions to a quantum description. Indeed the term "photon" was only coined later in 1928 after the initial work of Paul Dirac and the birth of quantum electrodynamics. The classical concept of radiative interaction was following quite a different itinerary since it was necessarily connected with the electromagnetic nature of light and phenomena such as scattering and radiative transfer as introduced by Subramanian Chandrasekhar earlier in the 50's and previously to the invention of the laser [3]. Chandrasekhar was interested in the study of the structure of stellar sources and therefore his formalism concerned other aspects of physics such as electrodynamics and statistics. But the main question we discuss here is around the concept of superposition of classical fields. The key contribution was done by Frits Zernike who in 1938 published a seminal paper on the fundamentals of partial coherence [4]. Zernike's theory was motivated by the search of optimized conditions of sources illuminating an optical micro-

\footnotetext{
${ }^{1}$ This article is dedicated to the memory of Prof. María Victoria Pérez Martín, full professor of optics, Faculty of Sciences, University of Santiago de Compostela and a pioneer in GRIN optics in Spain.
}

scope and providing images of phase objects. Indeed, the partial coherence is a key aspect to elucidate the optimized conditions for the resolving power of an image forming system, a rather practical aspect related to optical image quality. At an almost similar period of time the Dutch mathematician P. H. Van Cittert introduced the nowadays very well known theorem to define the degree of spatial coherence of a luminous source [5]. The contributions of Denis Gabor to the Information Theory appeared in nearly years $[6,7]$. During that period of years between the 30's and the 50's of the 20th $C$. the concept of measure of information and quantification of the same came to the scene in the field of information treatment and signal processing, with a particular interest on electronic signals. We cannot afford in this paper a complete description of all the relevant contributions, for obvious reasons and will concentrate on those aspects that later on contributed on a fundamental basis to the theory of partial coherence. These researches and activities had an expansion after the Second World War with a special mention of European research centers like the University of Manchester, in the United Kingdom; the Philips, in The Netherlands; the Institute of Optics, in France, and also the Bell Laboratories, in the United States with landmark contributions such as the mathematical theory of communication developed by Claude Shannon [8] did as well pioneering work.

The early studies on optical information processing, as developed in the 50's of the 20th C, had the particularity that the available sources had only at the time a weak degree of temporal and spatial coherence. The experiments, for example, designed and developed in the Physical Laboratories of the University of Manchester by Brian Thompson and Emil Wolf in 1956 were carried out in a classical diffractometer set-up and with the aim to analyze the structure of X-ray sources [9]. The experiment was giving a dramatic insight in the actual theoretical development of the partial coherence of light. Indeed, Emil Wolf published in Nature in 1953 a first seminal paper on 
a macroscopic theory of interference and diffraction of light by finite source [10]. The concept of spatial coherence of a luminous source was then introduced and extended based upon the former Van Cittert-Zernike theorem. Brian Thompson and Emil Wolf developed in 1956 the mentioned experimental setup with the aim to analyze the degree of spatial coherence of an extended source emitting under quasi-monochromatic conditions of radiation. The results were published in 1957 [9]. As explained by Emil Wolf himself [11], he received at the time of his post-doctoral position in Manchester, a proposal from Henry Lipson to revise the actual experiments they carried out in his laboratory for X-rays interference and diffraction by crystals. The observed patterns needed a deeper explanation that seemed to be related with the degree of partial coherence of the X-rays source. Brian Thompson and Emil Wolf initiated then a complete study by introducing the theoretical formulation in order to interpret the observed fringe patterns. An initial disagreement between the theory and the experimental results was then solved by recalibrating the masks used in the actual experimental set-up. The subsequently published results were indeed the first confirmation of the correctness of the Zernike's theory of partial coherence.

Since the early days of 1956 many other contributions on the Thompson-Wolf experiment have been published under various scopes and applications. The first interpretation of the experiment with the use of various He-Ne sources was done by Mario Bertolotti and collaborators. In 1965 they published a first study in which they examined the coherence properties of the laser He-Ne sources, under both single mode and multimode regime of the laser cavities of concave mirror type [12]. For the study they developed an experimental set-up based upon the Thompson-Wolf device. Later in 1966 M. Bertolotti and collaborators extended the previous study to the case of a two-mode laser beam [13]. The interference experiment was suitably modified by introducing a vibrating mirror to display the fringes from the slit in front of the detector. The field obtained by the superposition of the transverse modes $T E M_{30}$ and $T E M_{01}$ was then studied. From the obtained results it was concluded that the deviation of the field from Poisson statistics can be better measured through the beat interference term.

The pioneering experiments carried out on the 60's of the 20th $C$. have been since then revisited and modified to demonstrate the properties of the light sources under both the classical partial coherence theory and the coherence properties of sources and to measure the visibilities of second-order (onephoton) and fourth-order (two-photon) interference fringes in a Young's double-slit experiment slightly modified from the original Thompson-Wolf set-up. Inside these studies Saleh et al. established that the phenomena of diffraction, interference, and growth of coherence in conventional optics have their counterparts in bi-photon optics [14]. In a complementary article from Abouraddy et al. it was measured the degree of spatial coherence associated to non classical standard sources of radiation such as the light generated through the process of spontaneous parametric down conversion (SPDC) in a nonlinear crystal (NLC) [15]. As another example one can mention that the Thompson-Wolf experiment was applied to determine the spatial coherence in the Lau fringes experiment [16].
Owing to the characterization of spatial coherence of non standard classical sources of radiation the Thompson-Wolf experiment maintains nowadays its interest as a robust and flexible technique to be applied to the study of spatial coherence properties of a variety of radiation wavelength sources. One can mention the study on filamentary sources [17], light sources at soft-x-ray wavelengths, having the key aspect that the demonstration of nearly full spatial coherence has been one of the main goals of soft-x ray laser research $[18,19]$, electron interference [20], semiconductor emitters [21], to give an overview of current techniques and applied technologies interests.

The main motivation of the present article is to perform a revisited study of the Thompson-Wolf experiment by using two alternative laser sources, emitting under various visible wavelengths and having distinct resonant cavities geometries and laser beam quality. With this study we are reproducing the classical Thompson-Wolf experiment but with a certainly much higher degree of spatial coherence than the actual classical one for which thermal sources were considered. The paper is organized as follows: the first section is dedicated to the introduction; the second section is devoted to a revision of the classical definition of spatial coherence inside the context of the partial coherence theory. In the third section we describe the experimental set- up and the experimental results are presented in section fourth. The last section is dedicated to discussion and conclusions. We end the paper with the reference list.

\section{MATHEMATICAL FOUNDATION}

The theory of partial coherence can be formulated under two complementary frameworks: first, the traditional space-time domain in which we compare space-time signals by introducing a time shift between them; and second, in the spacefrequency domain [22], where we compare space-frequency signals with arbitrary spectral composition. In the present work we consider quasi-monochromatic fields.

The measurement of coherence is generally [23] carried out by using an interferometer that compares signals coming from two different space points and that introduces between them a time shift $\tau$.

If we use a Young-like interference experiment with scalar fields, the intensity at the screen can be expressed as

$$
I(\vec{r})=I^{1}(\vec{r})+I^{2}(\vec{r})+2 \sqrt{I^{1}(\vec{r})} \sqrt{I^{2}(\vec{r})} \Re\left\{\gamma\left(\overrightarrow{r_{1}}, \overrightarrow{r_{2}}, \tau\right)\right\},
$$

where $I^{1}(\vec{r})$ and $I^{2}(\vec{r})$ are the intensities produced by each of the pinholes alone and $\gamma\left(\overrightarrow{r_{1}}, \overrightarrow{r_{2}}, \tau\right)$ is the so-called complex degree of coherence (CDC). This quantity is related to the mutual coherence function (MCF), $\Gamma\left(\overrightarrow{r_{1}}, \overrightarrow{r_{2}}, \tau\right)=\left\langle U\left(\overrightarrow{r_{1}}, t\right) * U\left(\overrightarrow{r_{2}}, t+\tau\right)\right\rangle$, and the intensities at each of the pinholes, $I\left(\overrightarrow{r_{1}}\right)$ and $I\left(\overrightarrow{r_{2}}\right)$, by

$$
\gamma\left(\overrightarrow{r_{1}}, \overrightarrow{r_{2}}, \tau\right)=\frac{\Gamma\left(\overrightarrow{r_{1}}, \overrightarrow{r_{2}}, \tau\right)}{\sqrt{I\left(\overrightarrow{r_{1}}\right)} \sqrt{I\left(\overrightarrow{r_{2}}\right)}} .
$$

Furthermore, in the case of quasi-monochromatic light, we can write

$$
\Re\left\{\gamma\left(\overrightarrow{r_{1}}, \overrightarrow{r_{2}}, \tau\right)\right\}=\left|\mu_{12}\right| \cos \left(\phi_{12}\right),
$$


where $\left|\mu_{12}\right|=\left|\gamma\left(\overrightarrow{r_{1}}, \overrightarrow{r_{2}}, 0\right)\right|$ is called the spectral degree of coherence (SDC).

The visibility of the fringes in Young's experiment is usually defined as [24]

$$
\mathcal{V}=\frac{I_{\max }-I_{\min }}{I_{\max }+I_{\min }}=2\left[\epsilon(r)+\frac{1}{\epsilon(r)}\right]^{-1}\left|\gamma\left(\overrightarrow{r_{1}}, \overrightarrow{r_{2}}, \tau\right)\right|
$$

with

$$
\epsilon(r)=\left[\frac{\left\langle I^{1}(\vec{r}, t)\right\rangle}{\left\langle I^{2}(\vec{r}, t)\right\rangle}\right]^{1 / 2} .
$$

Thus, when the two intensities from the pinholes are equal, the visibility of the fringes is equal to the CDC between the two pinholes.

The so called Thompson-Wolf (TW) experiment is a modification of the Young's double slit experiment. In this experiment, a secondary source is placed at the focal point of a lens, which has after it a mask with two identical pinholes and a second lens which can be similar to the first one. The detector is placed in the back focal plane of this second lens. Essentially, TW is a diffractometer (see Figure 1).

Originally [9], the experiment was performed with a mercury-vapor compact source lamp with the yellow doublet $(\lambda=579 \mathrm{~nm})$ selected; i.e. with an inhomogeneous, quasi-monochromatic source of light. This had the advantage of reducing the problem of propagation of the MCF from the source to the mask to a single, two-dimensional spatial Fourier transform of the spatial intensity distribution by means of the Van Cittert-Zernike theorem (see, e.g., [25]) in the scalar case and planar source approximation. The conjugate variables after the FT are the relative difference between the transversal coordinates of the two points (reduced coordinates $x_{12}, y_{12}$ ) divided by the wavelength and the focal of the lens $\left(\boldsymbol{\xi}=\mathbf{x}_{12} / \vec{\lambda} f\right.$ and $\left.\boldsymbol{\eta}=\mathbf{y}_{12} / \vec{\lambda} f\right)$; therefore, the MCF between the two pinholes $\left(\overrightarrow{r_{1}}, \overrightarrow{r_{2}}\right)$ can be pictured as the complex amplitude at some point in a certain diffraction pattern [26].

However, the scalar description of the TW experiment using the MCF usually falls short when the polarization properties of the fields cannot be neglected. In these cases one may use the statistical electromagnetic fields formalism.

To a first order approximation, we can study [24] the secondorder coherence properties of an electromagnetic beam by means of the $2 \times 2$ cross spectral density matrix $\overleftrightarrow{W}$ (CSDM) with the elements

$$
W_{i j}(\vec{\rho}, \vec{\rho}, v)=\left\langle E_{i}^{*}(\vec{\rho}, v) E_{j}(\vec{\rho}, v)\right\rangle,
$$

where each of the components $E_{i}(\vec{\rho}, v)$ with $i=x, y$ represents one of the Cartesian components of the transverse electric field of the beam in the frequency domain.

This description holds only if we can ignore the contributions to the field derived from the presence of a third Cartesian component of the electric field [27], but given that the object under study is a (laser) beam, it is reasonable to neglect the contribution of this longitudinal component.
The propagation of the CSDM is given by a generalization of the Sommerfeld-Rayleigh formula [26],

$$
\begin{aligned}
& \overleftrightarrow{W}\left(\overrightarrow{r_{1}}, \overrightarrow{r_{2}}, \omega\right)= \\
& \iint \mathbf{K}_{\infty}^{+}\left({\overrightarrow{\rho_{1}}}^{1}, \overrightarrow{r_{1}}, \omega\right) \circ \overleftrightarrow{W}_{\perp}^{(0)}\left(\overrightarrow{\rho_{1}}, \overrightarrow{\rho_{2}}, \omega\right) \circ \mathbf{K}_{\infty}\left(\overrightarrow{\rho_{2}}, \overrightarrow{r_{2}}, \omega\right) d^{2} \overrightarrow{\rho_{1}} d^{2} \overrightarrow{\rho_{2}}
\end{aligned}
$$

where $\circ$ denotes matrix multiplication,

$$
\mathbf{K}_{\infty}(\vec{\rho}, \vec{r}, \omega)=\frac{1}{\lambda}\left[\begin{array}{ccc}
-s_{z} & 0 & s_{x} \\
0 & -s_{z} & s_{y}
\end{array}\right] \frac{e^{i k|\vec{r}|}}{|\vec{r}|} e^{-i k \vec{\rho} \cdot \vec{s}}
$$

is the propagator of the $\omega$ component of the field from $\vec{\rho}$ to $\vec{r}$ in the far field approximation; and $\overleftrightarrow{W}_{\perp}^{(0)}\left(\vec{\rho}_{1}, \overrightarrow{\rho_{2}}, \omega\right)$ is the transversal component of the CSDM

$$
\overleftrightarrow{W}_{\perp}=\left[\begin{array}{ll}
W_{x x} & W_{x y} \\
W_{y x} & W_{y y}
\end{array}\right]
$$

If we assume the paraxial approximation, $s_{x}, s_{y}=0$, we can neglect the contribution due to the longitudinal component of the propagated field $E_{z}$. Therefore we will consider only $2 \times 2$ CSDMs because of the transversal nature of the beam in the paraxial regime, and we may rewrite the propagators as

$$
\mathbf{K}_{\perp}(\vec{\rho}, \vec{r}, \omega)=\frac{1}{\lambda}\left[\begin{array}{cc}
-s_{z} & 0 \\
0 & -s_{z}
\end{array}\right] \frac{e^{i k|\vec{r}|}}{|\vec{r}|} e^{-i k \vec{\rho} \cdot \vec{s}} .
$$

The polarization properties of laser beams have been described recently [28]. If we suppose a laser beam with an arbitrary number of laser modes, the coherent-mode representation [29] for the diagonal tems of the CSDM is given by

$$
\begin{aligned}
W_{x x}\left(\overrightarrow{\rho_{1}}, \overrightarrow{\rho_{2}}, \omega\right) & =\sum_{n=1}^{\infty} \lambda_{n}(\omega) \phi_{n}^{*}\left(\overrightarrow{\rho_{1}}, \omega\right) \phi_{n}\left(\overrightarrow{\rho_{2}}, \omega\right) \\
& =W_{y y}\left(\overrightarrow{\rho_{1}}, \overrightarrow{\rho_{2}}, \omega\right),
\end{aligned}
$$

where the functions $\phi_{n}(\vec{\rho}, \omega)$ are identical with the laser resonator modes of the monochromatic scalar theory of Fox and $\mathrm{Li}$ (see [28]), and the last equality holds if we assume that the cavity is rotationally invariant.

The off-diagonal terms can be expanded as

$$
\begin{aligned}
W_{x y}\left(\overrightarrow{\rho_{1}}, \overrightarrow{\rho_{2}}, \omega\right) & =W_{y x}\left(\overrightarrow{\rho_{2}}, \overrightarrow{\rho_{1}}, \omega\right) \\
& =\sum_{n=1}^{\infty} \sum_{m=1}^{\infty} \Lambda_{n m}(\omega) \phi_{n}^{*}\left(\overrightarrow{\rho_{1}}, \omega\right) \phi_{m}\left(\overrightarrow{\rho_{2}}, \omega\right) .
\end{aligned}
$$

From Eqs. (11) and (13) we can see that the elements of the CSDM between two points with position vectors $\overrightarrow{\rho_{1}}$ and $\overrightarrow{\rho_{2}}$ can be decomposed as a sum of products of functions that depend only on one of the position vectors.

Using the same procedure as Thompson and Wolf, we will now derive an expression for the visibility of the intensity profile at the back focal plane of a diffractometer (see Figure 1).

Aimed with the knowledge of the CSDM at the output plane of the laser at $z=0, \overleftrightarrow{W}(0)$, we can use the propagation law 


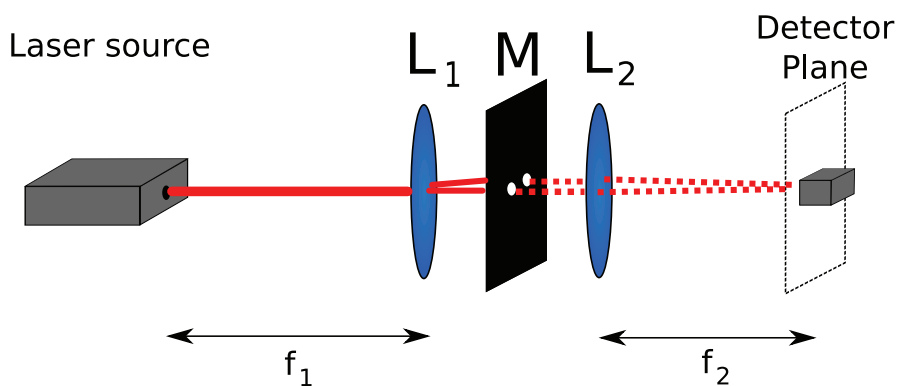

FIG. 1 Schematic diagram of the experimental set-up. Laser source: we used two alternative sources: He- $\mathrm{Ne}(\lambda=632.8 \mathrm{~nm})$ with $40 \mathrm{~mW}$ of exit power and a green laser pointer $(\lambda=532 \mathrm{~nm})$ with $50 \mathrm{~mW}$ exit power, respectively. $L_{1}$ : Collimating lens with focal distance $f_{1}=100 \mathrm{~cm}$. $L_{2}$ : Convergent lens with focal distance $f_{2}=50 \mathrm{~cm} . M$ : mask with the double slit. Two cases studied: rectangular slits $(0,5 \mathrm{~mm}$ width, 1,5 mm separation) and circular ones (0,5 $\mathrm{mm}$ diameter, $2 \mathrm{~mm}$ separation). Detection plane: A Spiricon system was used. (See text for details).

(see Eq. (7)) to obtain the CSDM at the points $\overrightarrow{r_{1}}=\left(x_{1}, y_{1}, f_{1}\right)$ and $\overrightarrow{r_{2}}=\left(x_{2}, y_{2}, f_{1}\right)$ of $L_{1}$ :

$$
\begin{aligned}
& \overleftrightarrow{W}\left(\overrightarrow{r_{1}}, \overrightarrow{r_{2}}, \omega\right) \\
& =\iint \mathbf{K}_{\perp}^{\dagger}\left({\overrightarrow{\rho_{1}}}^{1}, \overrightarrow{r_{1}}, \omega\right) \circ \overleftrightarrow{W}_{\perp}^{(0)}\left(\overrightarrow{\rho_{1}}, \overrightarrow{\rho_{2}}, \omega\right) \circ \mathbf{K}_{\perp}\left(\overrightarrow{\rho_{2}}, \overrightarrow{r_{2}}, \omega\right) d^{2}{\overrightarrow{\rho_{1}}}^{2}{\overrightarrow{\rho_{2}}}^{(15)} \\
& =B \frac{1}{\left(\lambda f_{1}\right)^{2}} \iint \overleftrightarrow{W}(0) \exp \left[\frac{i k}{f_{1}}\left(\rho_{2} r_{2 \perp}-\rho_{1} r_{1 \perp}\right)\right] d^{2}{\overrightarrow{\rho_{1}}}^{2}{\overrightarrow{\rho_{2}}}
\end{aligned}
$$

where the subscript $\perp$ stands for the fact that we are only taking into account the transversal part of the vector. Therefore, as the integration is performed over the spatial extension of the source, we will effectively obtain the spatial Fourier transform of the original CSDM at $L_{1}$. Furthermore, as we know that the terms of the CSDM is a sum of separable components at $L_{1}$,

$$
\begin{aligned}
& W_{x x}\left(\overrightarrow{r_{1}}, \overrightarrow{r_{2}}, \omega\right)=W_{y y}\left(\overrightarrow{r_{1}}, \overrightarrow{r_{2}}, \omega\right) \\
& =B \frac{1}{\left(\lambda f_{1}\right)^{2}} \sum_{n=1}^{\infty} \lambda_{n}(\omega) \mathcal{F}\left[\phi_{n}^{*}\right]\left(\overrightarrow{r_{1}} / \lambda f, \omega\right) \mathcal{F}\left[\phi_{n}\right]\left(\overrightarrow{r_{2}} / \lambda f, \omega\right)
\end{aligned}
$$

and

$$
\begin{aligned}
& W_{x y}\left(\overrightarrow{\rho_{1}}, \overrightarrow{\rho_{2}}, \omega\right)=W_{y x}\left(\overrightarrow{\rho_{2}}, \overrightarrow{\rho_{1}}, \omega\right) \\
& =B \frac{1}{\left(\lambda f_{1}\right)^{2}} \sum_{n=1}^{\infty} \sum_{m=1}^{\infty} \Lambda_{n m}(\omega) \mathcal{F}\left[\phi_{n}^{*}\right]\left(\overrightarrow{r_{1}} / \lambda f, \omega\right) \mathcal{F}\left[\phi_{m}\right]\left(\overrightarrow{r_{2}} / \lambda f, \omega\right) .
\end{aligned}
$$

If the mask is placed very close to the first lens, we can assume $[9,22]$ that the corresponding coordinates for the transversal components of the points in the mask and those of the lens are the same. Then, just after the mask with transmitance $M(r)$, we have

$$
\overleftrightarrow{W}^{\prime}\left(\overrightarrow{r_{1}}, \overrightarrow{r_{2}}, \omega\right)=M^{*}\left(\overrightarrow{r_{1}}\right) M\left(\overrightarrow{r_{2}}\right) \overleftrightarrow{W}\left(\overrightarrow{r_{1}}, \overrightarrow{r_{2}}, \omega\right)
$$

If the mask consists on two identical pinholes at the points $\vec{r}_{1}{ }^{\prime}$ and ${\overrightarrow{r_{2}}}^{\prime}$ respectively, we can choose the appropriate points to perform the interference, and then we can apply Eq. (1) to get the visibility. According to Eq. (4), the maximum visibility is obtained when the mask is positioned in such a way that the intensity at the two pinholes is the same. In this situation we get for the spectral degree of coherence [28]

$$
\begin{aligned}
& \left|\mu_{12}\right|=\left|\frac{\operatorname{Tr} \overleftrightarrow{W}\left({\overrightarrow{r_{1}}}^{\prime},{\overrightarrow{r_{2}}}^{\prime}, \omega\right)}{\sqrt{\operatorname{Tr} \overleftrightarrow{W}\left({\overrightarrow{r_{1}}}^{\prime},{\overrightarrow{r_{1}}}^{\prime}, \omega\right)} \sqrt{\operatorname{Tr} \overleftrightarrow{W}\left({\overrightarrow{r_{2}}}^{\prime},{\overrightarrow{r_{2}}}^{\prime}, \omega\right)}}\right| \\
& =\mid \frac{\sum \lambda_{n}(\omega) \mathcal{F}\left[\phi_{n}^{*}\right]\left(\overrightarrow{r_{1}} / \lambda f, \omega\right) \mathcal{F}\left[\phi_{n}\right]\left(\overrightarrow{r_{2}} / \lambda f, \omega\right)}{\left(\begin{array}{c}
\sqrt{\sum \lambda_{n}(\omega) \mathcal{F}\left[\phi_{n}^{*}\right]\left(\overrightarrow{r_{1}} / \lambda f, \omega\right) \mathcal{F}\left[\phi_{n}\right]\left(\overrightarrow{r_{1}} / \lambda f, \omega\right)} \\
\times \sqrt{\sum \lambda_{n}(\omega) \mathcal{F}\left[\phi_{n}^{*}\right]\left(\overrightarrow{r_{2}} / \lambda f, \omega\right) \mathcal{F}\left[\phi_{n}\right]\left(\overrightarrow{r_{2}} / \lambda f, \omega\right)}
\end{array}\right)},
\end{aligned}
$$

which equals the visibility of the fringe pattern at the back focal plane.

\section{EXPERIMENTAL SETUP}

As in [9], we used a diffractometer like the one depicted in Figure 1 to measure the degree of spatial coherence of two different laser beams.

The laser sources were placed at the object focal plane of the first convergent lens, $\mathrm{L}_{1}$, with focal length of $100 \mathrm{~cm}$. Immediately after it the mask was placed. We used two types of masks throughout the experiment: one with two vertical, parallel slits of width $0.05 \mathrm{~cm}$ and a separation of $0.15 \mathrm{~cm}$, and another one with two pinholes with the same width as in the double-slit mask, and a separation of $0.2 \mathrm{~cm}$. The thicknesses of the masks were much smaller than the diameter of the hole to assure a nearly 2-dimensional diffraction screen. They were constructed in aluminium with a dark coating. After the mask, we placed a second convergent lens, $\mathrm{L}_{2}$, with focal length $50 \mathrm{~cm}$ and a Spiricon SP620U beam profiler to record the fringe pattern at the back focal plane. Both lenses were aberration-free.

We used two different laser sources: a commercial He-Ne gas laser (JDS-Uniphase) emitting approximately $40 \mathrm{~mW}$ of power at $632.8 \mathrm{~nm}$ with a gaussian shaped beam, and a green laser pointer (Lasing, S.A.) emitting $50 \mathrm{~mW}$ of power with a wavelength of $532 \mathrm{~nm}$.

As the power of both lasers could damage the active surface of the beam profiler, we used three neutral filters to reduce the amount of light falling on to it. In the experiment, care was taken to assure a correct alignment of the two pinholes (or slits) with respect to the laser beam incident point in the mask.

\section{RESULTS}

The captured images were selected among those recorded by the system so as to show the maximum contrast in the actual experiment. Then, we obtained the contributions due to each one of the holes as a complementary test of the fringes visibility. In the case of the He-Ne laser, the maximum visibility was obtained when the intensities produced by each of the holes alone were similar, while in the case of the green laser pointer the maximum visibility was achieved with an asymmetry in the single-hole diffracted intensity distribution. 

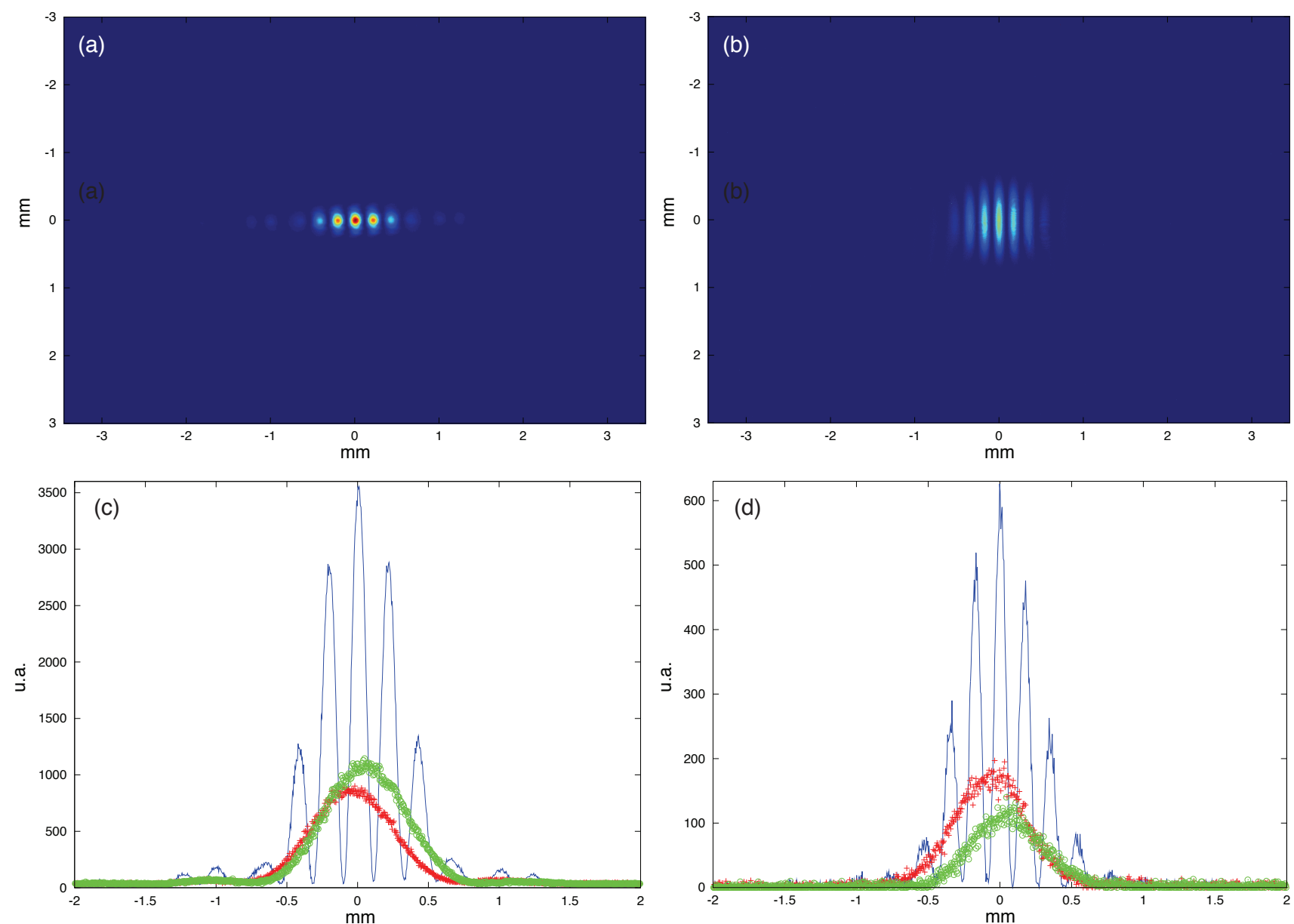

FIG. 2 The captured images of the interference patterns by the Spiricon system and a He-Ne laser source $(\lambda=632.8 \mathrm{~nm}$ ) and $40 \mathrm{~mW}$ power: (a) and (b), and corresponding line profiles (c) and (d), respectively. (a) Two-slits mask (width: $0.05 \mathrm{~cm}$, separation: $0.15 \mathrm{~cm}$ ). (b) Two-pinholes mask (width: $0.05 \mathrm{~cm}$, separation: $0.2 \mathrm{~cm}$ ). (c) Line profile of the image as in (a) recording. Green curve corresponds to the recording with a single slit (centered). Red line corresponds to the recording of the other slits. Slight decentration corresponds to the slits separation. For convenience, in (c) and (d) arbitrary units were used.

Figures 2 and 3 display the experimental results obtained with the experimental set-up as shown in Figure 1. Figure 2 shows the experimental results obtained with the calibrated $\mathrm{He}-\mathrm{Ne}$ laser used as a probe beam and Figure 3 shows the results with the green laser whose modal regime was unknown.

A comparison of the results of the two-slits and the twopinholes interference patterns, respectively, indicates that the green laser pointer exhibited a modal regime which was not compatible with a single-mode regime as in the He-Ne probe beam, as its associated degree of spatial coherence is not unitary.

\section{DISCUSSION AND CONCLUSIONS}

We have revisited the Thompson-Wolf experiment with the aim to obtain comparative results with two different lasers sources. The lasers operated under different modal regime. According to previous results obtained by Bertolotti et al [13], it is observed that lasers emitting with a regime other than single mode produce interference fringes with lower visibitlity that the one expected for a monomode regime. In the actual case, the line profile associated to the interference term exhibits an asymmetry contrary to the line profile obtained for the monomode regime. Moreover, the diffracted field associated with each one of the pinholes, as detected separately, shows line profiles that are non-gaussians if the arbitrarymode beam illuminates the pinhole.

As in [28], and as it was pointed out by Bertolotti experimentally, if we have a single-mode laser, the visibility of the fringes will approach unity, as can be seen if we particularize Eq. (20) for a sum with a single term. However, if each of the pinholes is illuminated by more than one mode, the visibility of the fringes will be reduced.

The results indicate that the Thompson-Wolf experiment is sensible to the modal regime of the laser source and could be used for the purpose of controlling the modal structure of the source. A final point to mention is that the TW experiment carried out by us needed only unexpensive infrastructure and could be implemented for the purpose of teaching demonstrations.

\section{ACKNOWLEDGEMENTS}

The financial support of the Spanish Ministry of Science and Innovation (MICINN), grant TEC2008-04105 is acknowl- 

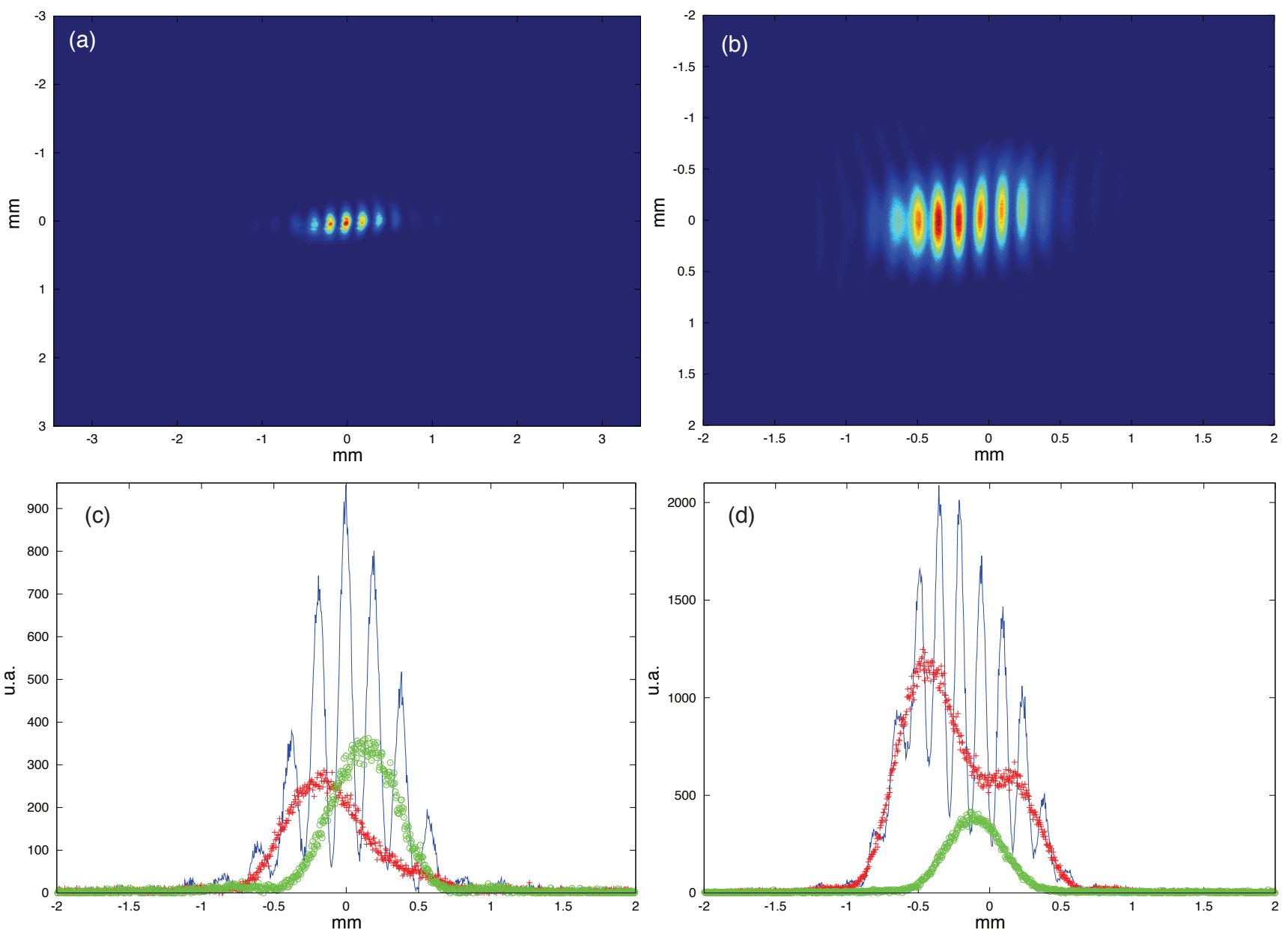

FIC. 3 The captured images of the interference patterns by the Spiricon system and a green pointer laser source $(\lambda=532 \mathrm{~nm})$ and $50 \mathrm{~mW}$ power: (a) and (b), and corresponding line profiles (c) and (d), respectively. (a) Two-slits mask (width: $0.05 \mathrm{~cm}$, separation: $0.15 \mathrm{~cm}$ ). (b) Two-pinholes mask (width: $0.05 \mathrm{~cm}$, separation: $0.2 \mathrm{~cm}$ ). (c) Line profile of the image as in (a) recording. Green curve corresponds to the recording with a single slit (centered). Red line corresponds to the recording of the other slits. Slight decentration corresponds to the slits separation. For convenience, in (c) and (d) arbitrary units were used. (See text for details).

edged. We are indebted to Maria Paz Hernández-Garay, Ana Manzanares and Oscar Martínez-Matos for their very valuable support and suggestions.

\section{References}

[1] A. Einstein, "Zur Quantentheorie der strahlung" Phys. Z. 18, 121 (1917) in german.

[2] D. ter Haar, On the Quantum Theory of Radiation, The Old Quantum Theory (Pergamon Press, New York, 1967).

[3] S. Chandrasekhar, Radiative Transfer (New York, Dover, 1960).

[4] F. Zernike, "Phase contrast, a new method for the microscopic observation of transpsrent objects" Physica 9, 686 (1938).

[5] P. H. van Cittert, "Die Wahrscheinliche Schwingungsverteilung in Einer von Einer Lichtquelle Direkt Oder Mittels Einer Linse Beleuchteten Ebene" Physica 1, 201 (1934).

[6] D. Gabor, "Theory of communication" J. Inst. Elect. Eng. 93429 (1949).

[7] D. Gabor, "Optical Transmission" in Proceedings of the Third London Conference on Information Theory C. Cherry, ed. (Butterworth Scientific Publications, London, 1956).

[8] C. E. Shannon, "A mathematical theory of communication" Bell
Syst. Tech. J. 27, 379 and 623, (1948).

[9] B. Thompson, and E. Wolf, "Two-Beam Interference with Partially Coherent Light" J. Opt. Soc. Am. 47, 895 (1957).

[10] E. Wolf, "A Macroscopic Theory of Interference and Diffraction of Light from Finite Sources" Nature 172, 535 (1953).

[11] E. Wolf, "Early days of coherence theory and the First Rochester Conference on coherence" in A Jewel on the Crown - 75th Anniversary Essays, C. R. Stroud Jr. ed. (The Institute of Optics, University of Rochester, 2004)

[12] M. Bertolotti, B. Daino, F. Gori, and D. Sette, "Coherence properties of a laser beam" Nuovo Cimento 38, 1505 (1965).

[13] M. Bertolotti, B. Crosignani, P. Di Porto, and D. Sette, "Coherence and statistical properties of a two-mode laser beam" Phys. Rev. 150, 1054 (1966).

[14] B. E. A. Saleh, A. F. Abouraddy, A. V. Sergienko, and M. C. Teich, "Duality between partial coherence and partial entanglement" Phys. Rev. A 62, 043816 (2000).

[15] A. F. Abouraddy, B. E. A. Saleh, A. V. Sergienko, and M. C. Teich, "Double-slit interference of biphotons generated in spontaneous parametric downconversion from a thick crystal" J. Opt. B: Quantum S. 0. 3, 550 (2001).

[16] S. Chitralekha, K. V. Avudainayagam, and S. V. Pappu, "Role of spatial coherence on the rotation sensitivity of Lau fringes: an ex- 
perimental study" Appl. Opt. 28, 345 (1989).

[17] M. E. Mancenido, G. Pozzi, L. Zunino, and M. Garavaglia, "Young interferential experiments with filamentary light sources" Proc. SPIE 3190, 290 (1997).

[18] Y. Liu, M. Seminario, F. G. Tomasel, C. Chang, J. J. Rocca, and D. T. Attwood, "Achievement of essentially full spatial coherence in a high-average-power soft-x-ray laser" Phys. Rev. A 63, 033802 (2001).

[19] K. M. Rosfjord, Y. Liu, and D. T. Attwood, "Tunable Coherent Soft X-Rays" IEEE J. Sel. Top. Quant 10, 1405 (2004).

[20] H. Lichte, "Electron interference: mystery and reality" Philos. T. R. Soc. Lond., 360, 897 (2002).

[21] F. J. Duarte, "Coherent electrically excited organic semiconductors: coherent or laser emission?" Appl. Phys. B 90, 101 (2008).

[22] E. Wolf, "A Macroscopic Theory of Interference and Diffraction of Light from Finite Sources. I. Fields with a Narrow Spectral Range" P. Roy. Soc. A-Math. Phy. 255, 1160 (1954).

[23] M. Bass, C. DeCusatis, J. Enoch, V. Lakshminarayanan, G. Li,C. Mac- donald, V. Mahajan, and E. Van Stryland, Handbook of Optics, Third Edition, Volume I: Geometrical and Physical Optics, Polarized Light, Components and Instruments(set) (MCGraw-Hill, Inc., USA, 2010).

[24] L. Mandel, and E. Wolf, Optical Coherence and Quantum Optics (Cambridge University Press, Cambridge, 1995).

[25] A. S. Marathay, Elements of Optical Coherence Theory (John Wiley at Sons, 1982)

[26] M. A. Alonso, 0. Korotkova, and E. Wolf, "Propagation of the electric correlation matrix and the van Cittert-Zernike theorem for random electromagnetic fields" J. Mod. Opt. 53, 7 (2006).

[27] T. Setälä, A. Schevchenko, M.Kaivola, and A. T. Friberg, "Degree of polarization for optical near fields" Phys. Rev. E 66, 016615 (2002).

[28] E. Wolf, "Coherence and polarization properties of electromagnetic laser modes" Opt. Commun. 265, 60 (2006)

[29] K. Kim, and E. Wolf, "A scalar-mode representation of stochastic, planar, electromagnetic sources" Opt. Commun. 261, 19 (2006). 